\section{Stichwort Lebensqualität}

Mit zunehmendem Lebensalter steigt die Inzidenz maligner Erkrankungen, gleichzeitig leben Krebspatienten dank moderner Therapien immer länger mit stabiler Krankheit. Verändert sich die Lebensqualität abhängig vom Alter?

\begin{abstract}
A nhand der Daten von 25 randomisierten, kontrollierten Phase-III-Studien der European Organization for Research and Treatment of Cancer (EORTC) wurde untersucht, wie sich maligne Erkrankungen auf die Lebensqualität auswirken und ob es dabei eine Altersabhängigkeit gibt. In der Krebskohorte wurden Patienten mit folgenden Indikationen eingeschlossen: Kolorektal-, Lungen-, Ösophagus-, Ovarial-, Prostata-, Hoden-, Mamma-, Pankreaskarzinom, Kopf-Hals-Tumoren, Melanom.

In allen Studien war die Lebensqualität als sekundärer Endpunkt mithilfe des EORTC QLQ-C30 ermittelt worden. Berücksichtigt wurden nur vollständig ausgefüllte Fragebögen (6.024 Patienten aus 34 Ländern). Manche Patienten hatten vor Studienbeginn bereits eine
\end{abstract}

Krebstherapie erhalten (Operation, palliative Chemotherapie oder Strahlentherapie), andere nicht. Verglichen wurden die mittleren EORTC-QLQ-C30-Scores der Krebskohorte mit denen von 5 bevölkerungsbasierten Kohorten. 3 Alterskategorien wurden gebildet: $<50$ Jahre, 50-70 Jahre und > 70 Jahre. Eine Differenz von mindestens 10 Punkten auf der hundertstufigen Skala galt als klinisch bedeutsam.

Hinsichtlich ihrer Lebensqualität hatten die Krebspatienten grundsätzlich schlechtere Werte als die bevölkerungsbasierte Kohorte. Allerdings variierten bestimmte Lebensqualitäts-Domänen mit dem Lebensalter. Jüngere Krebspatienten hatten im Vergleich zur bevölkerungsbasierten Kohorte häufiger finanzielle Probleme sowie größere soziale
Schwierigkeiten. Ältere Krebspatienten litten häufiger unter Appetitlosigkeit. Mit steigendem Lebensalter verschlechterte sich die Lebensqualität bezüglich physischer Funktionen und Obstipation, sie verbesserte sich jedoch hinsichtlich sozialer Funktionen, Schlafstörungen und finanzieller Problemen (alle $\mathrm{p}<0,05)$.

Fazit: Die Lebensqualität war infolge von Krebserkrankungen eingeschränkt - wie stark und in welcher Form, hing vom Alter ab. Manche Komponenten waren bei älteren Krebspatienten besser, andere schlechter. Bei der Betreuung der Patienten müsse, so die Interpretation, auf altersspezifische Bedürfnisse und Probleme geachtet werden.

Kathrin von Kieseritzky

Quinten $C$ et al. The effects of age on health-related quality of life in cancer populations: A pooled analysis of randomized controlled trials using the European Organisation for Research and Treatment of Cancer (EORTC) QLQ-C30 involving 6024 cancer patients. Eur J Cancer. 2015;51(18):2808-19.

\title{
E-Monitoring von Symptomen
}

\section{Kann elektronisches Monitoring von Symptomen und klinischen Syndromen zusätzlich zur onkologischen Visite die Betreuung von Patienten mit malignen Erkrankungen verbessern? Diese Frage wurde in einer Phase-III-Studie gestellt.}

P atienten mit unheilbaren malignen Erkrankungen erleben im Krankheitsverlauf vielgestaltige, wechselnde Symptome, die Befinden und Lebensqualität beeinträchtigen können, oft aber nicht oder erst spät erkannt werden. In einer Phase-III-Studie wurde postuliert, dass ein Real-Time-Monitoring von Symptomen und klinischen Syndromen die Betreuung und damit die Situation der Patienten erheblich verbessern kann. Um das Real-Time-Monitoring zu realisieren, wurde das sogenannte E-MOSAIC („electronic monitoring of symptoms and syndromes associated with cancer") angewendet. Folgende Aspekte werden hier berücksichtigt: EdmontonSymptom-Assessment-Scale, $\leq 3$ zusätzliche Symptome, geschätzte Nahrungsaufnahme, Gewichtsänderungen, Kar-
nofsky-Performancestatus, Schmerzmedikation, Medikation für Fatigue und Unterstützung der Ernährung.

An der Studie beteiligten sich 8 onkologische Zentren mit 82 Onkologen und 264 Patienten mit unheilbaren soliden Tumoren, die eine palliative Chemotherapie (meist Platin und Taxane, gefolgt von Pyrimidinen) erhielten. Unmittelbar vor der wöchentlichen onkologischen Visite füllten die Patienten der Interventionsgruppe den E-MOSAIC aus. Ermittelt wurde die Differenz in der globalen Lebensqualität (G-QoL; 29/30 Items des EORTC-QlQ-C30) zwischen Studienbeginn und Woche 6. Die Intention-to-treat-Analyse (ITT) umfasste die Daten aller Patienten.

Zwischen Interventions- und Kontrollgruppe betrug die Differenz im
G-QoL 6,8 Punkte $(\mathrm{p}=0,11)$, mit einem nicht signifikanten Vorteil für die Interventionsgruppe. In einer Sensitivitätsanalyse (Onkologen, die mindestens $2 \mathrm{~Pa}$ tienten behandelten) betrug die Differenz 8,96 Punkte $(p=0,07)$. Die erhoffte Verbesserung der globalen Lebensqualität trat somit nur begrenzt ein. Die ITT-Analyse zeigte, dass in der Interventionsgruppe mehr Patienten mit starken Beschwerden eine unmittelbare Therapie erhielten.

Fazit: Enges Monitoring und die Behandlung von Symptomen und klinischen Syndromen verbessert die Beschwerden von Krebspatienten, nicht jedoch die globale Lebensqualität. Den Forschern zufolge könnte ein Real-TimeMonitoring im onkologischen Routineablauf dennoch vorteilhaft sein.

Kathrin von Kieseritzky

Strasser F et al. The effect of real-time electronic monitoring of patient-reported symptoms and clinical syndromes in outpatient workflow of medical oncologists: E-MOSAIC, a multicenter cluster-randomized phase III study (SAKK 95/06). Ann Oncol. 2016; 27(2):324-32. 